\title{
Integração do Modelo MGB-IPH com Sistema de Informação Geográfica
}

\author{
Fernando Mainardi Fan*, Walter Collischonn* \\ fernando.fan@ufrgs.br; collischonn@iph.ufrgs.br
}

Recebido:18/08/12 - revisado: 21/05/13 - aceito: 30/10/13

\section{RESUMO}

A modelagem hidrológica é uma ferramenta extremamente útil para a realização de avaliações e apoio à tomada de decisão em questões relacionadas ao uso correto de recursos hídricos. Os modelos hidrológicos do tipo distribuído são capazes de representar o comportamento de uma bacia hidrográfica de forma complexa, permitindo análises e estudos detalhados a partir de seus resultados. Um dos requisitos básicos para o uso destes modelos distribuídos é a descrição fisiográfica detalhada da bacia estudada, obtida através do uso de plataformas de Sistemas de Informação Geográfica (SIG). Visando potencializar o uso da simulação hidrológica e o desenvolvimento de soluções para o correto gerenciamento de recursos hídricos, o presente trabalho apresenta a concepção de um acoplamento do tipo rígido entre a plataforma de SIG MapWindow GIS® e o modelo hidrológico MGB-IPH. A ferramenta concebida foi testada com sucesso na modelagem da bacia hidrográfica do rio Ijuí. A aplicação demonstrou que um grau elevado de integração entre o modelo hidrológico e o SIG foi obtido. Foram criadas ferramentas úteis para manipulação e representação simplificada dos dados de entrada no ambiente SIG, e para pósprocessamento e análise dos resultados do modelo hidrológico.

Palavras-chave: modelo hidrológico, sistema de informação geográfica, MGB-IPH

\section{INTRODUÇÃO}

Questões relacionadas a problemas de falta de água e uso correto dos recursos hídricos estão entre os grandes desafios a serem enfrentados pela humanidade nas próximas décadas. Para que estas demandas sejam sobrepujadas, a modelagem hidrológica se apresenta como uma das principais ferramentas a serem utilizadas, uma vez que é eficaz para a realização de previsões, estudos sobre efeitos de mudanças climáticas e de uso do solo, análises de disponibilidade de água e apoio à tomada de decisão, entre outras aplicações.

Os modelos hidrológicos são representações matemáticas do comportamento dos sistemas hídricos. Destacam-se, entre estes modelos, os modelos distribuídos de transformação chuva-vazão, que representam os processos de transformação da precipitação em escoamento e a propagação deste escoamento em uma bacia hidrográfica.

*Instituto de Pesquisas Hidráulicas - UFRGS
Para a simulação do comportamento de uma bacia hidrográfica, os modelos utilizam uma série de equações com dados de entrada diversos, como tipo e uso do solo na região estudada, precipitação ao longo do tempo, e outras variáveis climáticas. Como resultados, são geradas informações hidrológicas ao longo de vários pontos da bacia hidrográfica estudada, como vazão nos rios e balanços de água no solo.

Assim, a maior limitação da utilização de modelos hidrológicos distribuídos é a necessidade de manipulação de grandes quantidades de dados relacionados à variabilidade espacial e temporal dos parâmetros e variáveis que descrevem a heterogeneidade do comportamento hidrológico (TUCCI, 1998; PAIVA, 2009).

Os Sistemas de Informação Geográfica (SIG), com a sua capacidade de geração de bancos de dados espaciais e processamento das informações, são a ferramenta que possibilita a eliminação desta dificuldade de obtenção da descrição espacial detalhada da região de estudo. Dentre as variáveis comumente utilizadas como dados de entrada em modelos hidrológicos que podem ser fornecidas pelos SIG pode-se citar tipo e uso do solo, cobertura vegetal, relevo e distribuição de feições hidrológicas (MELO et al., 2008). 
Com suas raízes históricas nas disciplinas de cartografia computacional e processamento digital de imagens, os SIG são atualmente uma ferramenta altamente difundida para a solução de problemas que dependem de avaliações espaciais precisas e eficientes (MARTIN et al., 2005; SUI e MAGGIO, 1999).

Apesar do seu uso abrangente, a tecnologia SIG não foi especificamente desenvolvida para a modelagem de problemas de engenharia; inicialmente ela foi desenvolvida como uma ferramenta para armazenamento, manipulação, análise e apresentação de dados geográficos. Contudo, com o contínuo declínio do custo das tecnologias computacionais e o concomitante aumento na velocidade dos processadores, a tecnologia SIG foi disseminada entre os pesquisadores, desenvolvedores e usuários de modelos (MARTIN et al., 2005).

Tratando-se da área de recursos hídricos, por volta das décadas de 1970 e 1960, surgiram as primeiras ideias de se integrar os SIG aos estudos hidrológicos (MELO et al., 2008). Os primeiros esforços do uso de SIG se concentraram paralelamente em duas áreas: a aplicação da ferramenta para a obtenção de parâmetros de entrada para os modelos hidrológicos, e a utilização do SIG como interface para modelos de simulação (MARTIN et al., 2005).

O primeiro modelo hidrológico que se baseou em SIG para a obtenção de seus parâmetros foi o Topmodel (BEVEN e KIRKBY, 1979). Ele é um modelo de predição de vazão baseado na topografia obtida através de um MDE (Modelo Digital de Elevação). Desde então, muitos outros modelos têm sido desenvolvidos de alguma forma integrada aos SIG, buscando não única e necessariamente processar a modelagem dentro do SIG, mas sim uma melhoria na estimativa dos parâmetros dos modelos conceituais, a preparação das unidades espaciais para o uso de modelos distribuídos, a subdivisão automatizada da bacia em unidades menores, a apresentação espacial dos resultados e a simplificação da aplicação do modelo (MOORE et al., 1988; HESSION e SHANHOLTZ, 1988; OLIVIERIE et al., 1991; GOODCHILD et al., 1992; TIM et al., 1992; MOORE, 1996; FERRAZ et al., 1999; ZEILHOFER et al., 2003; WHITEAKER e MAIDMENT, 2004; COELHO, 2006).

Algumas integrações conhecidas e de uso atualmente difundido são as dos modelos SWAT (GEORGE e LEON, 2008; OLIVERA et al., 2006) e HEC-GeoHMS (USACE, 2009). Ambas utilizam um SIG para preparação dos dados de entrada e como interface de aplicação do modelo.
Segundo Goodchild (1993) e Mizgalewics e Maidment (1996) apud Zeihofer et al. (2003), existem três funções principais que são contribuições dos SIG à modelagem hidrológica:

I. Pré-processamento de dados espaciais: o SIG é utilizado para gerar informações espaciais de entrada no modelo hidrológico.

II. Suporte direto na modelagem: tarefas são executadas diretamente dentro do SIG, como ajustes e verificações.

III. Pós-processamento: os resultados do modelo hidrológico são trabalhados e visualizados dentro do SIG no seu contexto espacial.

Nyerges (1991) e Sui e Maggio (1999) sugerem que os SIG e os modelos hidrológicos podem ser integrados em três níveis de acoplamento, com diferentes estratégias de integração:

I. Acoplamento livre: O modelo funciona desvinculado ao SIG, que é usado apenas para pré, pós-processamento e visualização de feições espaciais e/ou resultados.

II. Acoplamento próximo ou rígido: $\mathrm{O}$ modelo funciona junto ao SIG, com todas as funções de geração, processamento e visualização funcionando através do SIG. Porém, a modelagem funciona de forma paralela, sendo o SIG necessário para que ela seja ativada (a interface do modelo é o SIG).

III. Acoplamento Pleno: As equações de modelagem hidrológica são programadas dentro do código do próprio SIG, ou uma estrutura de SIG é programada dentro do próprio modelo hidrológico. Esta forma de acoplamento possui, portanto, duas subdivisões.

A seguir, um breve relato sobre as vantagens e desvantagens de cada um dos métodos de acoplamento é realizado.

\section{Acoplamento livre ou solto}

O acoplamento livre entre SIG e modelos hidrológicos consiste na transferência de dados gerados através do SIG para serem utilizados como entrada no modelo. Após a simulação, os dados de saída tipicamente são transferidos para o SIG novamente para a apresentação dos resultados e realização de análises espaciais.

Este tipo de acoplamento é largamente adotado por pesquisadores devido ao desenvolvimento relativamente fácil (programação computacional é mínima). Outra vantagem desta abordagem é que 
programações redundantes podem ser evitadas. No entanto a conversão de dados entre os diferentes programas pode ser lenta e propensa a erros, e o acoplamento é limitado pela dependência do SIG e do modelo a formatos específicos de dados (SUI e MAGGIO, 1999).

Esta abordagem raramente permite aos usuários utilizar completamente as capacidades funcionais do SIG (i.é. ferramentas de análise espacial), já que o SIG é utilizado majoritariamente como uma ferramenta de visualização (MARTIN et al., 2005).

\section{Acoplamento próximo ou rígido}

No acoplamento próximo ou rígido, ao invés de transferências externas de arquivos os dados são trocados automaticamente entre o modelo hidrológico e o software SIG. Neste caso, a apresentação dos resultados do modelo é configurada como uma ferramenta interativa do SIG, e a preparação de dados de entrada e ativação do modelo é também feita através da interface do software (NYERGES, 1991; BURROUGH, 1990).

Esta abordagem aperfeiçoa o desempenho computacional e a interação entre os dois programas, traduzindo tudo para um ambiente de modelagem mais complexo (MARTIN et al., 2005).

\section{Acoplamento pleno}

O acoplamento pleno é a mais sofisticada forma de integração entre SIG e modelos hidrológicos. Ele é baseado na incorporação dos componentes funcionais de um sistema no outro, eliminando a necessidade de transferências intermediárias entre os softwares (LIAO e TIM, 1997).

O acoplamento pode consistir tanto na incorporação do SIG ao modelo hidrológico, como do modelo hidrológico ao SIG. O grande inconveniente do primeiro enfoque é que as funcionalidades de gerenciamento de dados e visualização dos softwares de modelagem hidrológica não são em absoluto comparáveis com os as capacidades disponíveis nos pacotes de softwares de SIG comerciais, além dos esforços de programação tenderem a ser intensos e muitas vezes redundantes (SUI e MAGGIO, 1999).

No segundo caso, o modelo hidrológico opera internamente ao software SIG, aproveitando as funcionalidades do mesmo para sobreposição de camadas de informação e gerenciamento espacial de variáveis. Devido à relativa complexidade envolvida no desenvolvimento destes sistemas, comumente os sistemas plenamente integrados utilizam equacionamentos simplificados e possuem funcionalidades limitadas em relação a operações como calibração automática (LIAO e TIM, 1997; SUI e MAGGIO, 1999; MARTIN et al., 2005).

Independentemente da forma de utilização dos SIG, é consenso que nos padrões atuais de modelagem hidrológica é impossível a utilização de um modelo distribuído sem que exista algum tipo de acoplamento a uma plataforma deste gênero.

Desta forma, visando potencializar o uso da simulação hidrológica, aprimorar a integração entre modelagem e as tecnologias SIG e promover desenvolvimento neste campo de estudo, o presente trabalho apresenta a concepção de um acoplamento entre a plataforma de SIG MapWindow GIS ${ }^{\circ}$ (AMES, 2006; AMES et al., 2008) e o modelo hidrológico MGB-IPH (COLLISCHONN e TUCCI, 2001).

\section{ACOPLAMENTO DO MGB-IPH AO MAP- WINDOW GIS $®$}

Neste trabalho, foi desenvolvido um acoplamento do tipo próximo (ou rígido), anteriormente descrito, em que os programas computacionais do modelo hidrológico e do SIG rodam de forma independente e são integrados através da troca interna de informações. Além disto, a execução do modelo hidrológico é realizada via interface desenvolvida dentro do SIG.

Esta forma de integração foi adotada para que as atividades de integração com o SIG não balizassem os desenvolvimentos paralelos do modelo hidrológico e da própria ferramenta de integração.

A seguir, são apresentadas as principais características dos materiais utilizados na construção desta integração, o MGB-IPH e o MapWindow GIS®.

\section{O modelo MGB-IPH}

O Modelo Hidrológico de Grandes Bacias, MGB-IPH, foi desenvolvido para simular o processo de transformação da chuva em vazão em grandes bacias hidrográficas (COLLISCHONN e TUCCI, 2001; COLLISCHONN et al., 2007). A versão original do modelo adotava uma subdivisão da bacia hidrográfica em elementos regulares (células quadradas), de cerca de 10 x $10 \mathrm{~km}$. Atualmente, é adotada no MGB-IPH uma discretização da bacia em unidades irregulares, definidas a partir de dados do relevo de um Modelo Digital de Elevação (MDE), e denominadas minibacias (GETIRANA et al., 2010; PEREIRA, 2010). 
Esta etapa de divisão da área de estudo em unidades menores, denominada discretização da bacia hidrográfica, é uma fase fundamental da aplicação de um modelo hidrológico distribuído. Existem vários métodos de discretização de bacias, que podem subdividir a área em unidades regulares (como quadrados e retângulos) ou unidades irregulares (tipicamente bacias menores) (PAZ et al., 2011). Esta subdivisão da bacia em minibacias, adotada nas versões mais recentes do modelo MGB-IPH é proveniente da aplicação das ferramentas disponibilizadas pelo pacote ArcHydro Tools, que é um pacote de ferramentas do software de SIG ArcGIS (MAIDMENT, 2002; ESRI, 2007; PAIVA, 2009; FAN et al., 2010a), ou da aplicação das ferramentas do TauDEM tools, que é um pacote presente no MapWindow GIS (TARBOTON, 2002).

Ainda, para a simulação hidrológica através do MGB-IPH, as minibacias que compõem a bacia hidrográfica são subdivididas em Unidades de Resposta Hidrológicas (URH ou HRU - Hydrological Response Unit), que são áreas de comportamento hidrológico similar. Considera-se que o comportamento hidrológico das URHs possa ser explicado por características físicas relacionadas aos solos (e.g. capacidade de armazenamento d'água, profundidade, porosidade, condutividade hidráulica) e a cobertura vegetal (e.g. índice de área foliar, interceptação, profundidade das raízes, albedo).

Além destas informações espaciais, para a aplicação do MGB-IPH, dados como informações fluviométricas e pluviométricas da bacia hidrográfica simulada devem ser obtidos externamente, processados, e então utilizados como entrada no modelo, e outras informações de parâmetros hidrológicos devem simplesmente ser preparadas pelo usuário do modelo.

O Quadro 01, seguinte, descreve de forma sumarizada os dados hidrológicos e climatológicos de entrada do modelo MGB-IPH.

Como um modelo-chuva vazão convencional, o principal resultado gerado pelo MGB-IPH é a vazão nas minibacias que compõem a área simulada. Contudo, outras informações sobre a região estudada também são geradas, como o balanço de água no solo, evapotranspiração diária e precipitação diária em cada minibacia.

O detalhamento das metodologias de cálculo dos processos hidrológicos no modelo MGB-IPH podem ser obtidas em Collischonn e Tucci (2001), Collischonn et al. (2007) e Paiva (2009).
Quadro 01 - Dados hidrológicos de entrada do modelo MGB-IPH.

\begin{tabular}{|l|l|}
\hline Dado de Entrada & Descrição \\
\hline Dados de Chuva & $\begin{array}{l}\text { Precipitação diária em pontos da } \\
\text { bacia hidrográfica em milíme- } \\
\text { tros de chuva. }\end{array}$ \\
\hline Dados de Vazão & $\begin{array}{l}\text { Informações diárias de vazão } \\
\text { provindas de estações fluviomé- } \\
\text { tricas na bacia hidrográfica. }\end{array}$ \\
\hline Dados de Clima & $\begin{array}{l}\text { Médias climáticas mensais dos } \\
\text { parâmetros Temperatura, Vento, } \\
\text { Umidade Relativa, Insolação e } \\
\text { Pressão da região de estudo. } \\
\text { Adicionalmente, podem ser } \\
\text { utilizados dados diários. }\end{array}$ \\
\hline
\end{tabular}

\section{A plataforma MapWindow GIS ${ }^{\circledR}$}

A plataforma MapWindow GIS® (AMES, 2006; AMES et al, 2008) é um programa livre que contém um grande número de funcionalidades apresentadas de forma simples, que geralmente são suficientes para satisfazer as necessidades básicas de um usuário de SIG. Nele, todas as ferramentas fundamentais de visualização e acoplamento com bases de dados, que caracterizam um SIG, estão presentes.

Por exemplo, um conjunto de ferramentas do MapWindow GIS® especialmente útil para o trabalho com recursos hídricos é o conjunto TauDEM Tools, que possibilitam o processamento de modelos digitais de elevação para a obtenção de informações hidrológicas, como a delimitação de bacias hidrográficas, de forma similar ao conjunto de ferramentas ArcHydro Tools.

Contudo, o maior aspecto do MapWindow GIS® é que ele não é limitado puramente às funcionalidades básicas disponíveis, pois ele permite que interfaces personalizadas sejam adicionadas ao programa através de plugins programados em diferentes linguagens de programação (AMES, 2006; AMES et al., 2008).

Um plugin pode ser definido como uma funcionalidade pré-programada que é utilizada para adicionar funções, ou ferramentas, ao programa no qual ele é instalado. No caso do MapWindow GIS®, os plugins consistem em programas compilados como bibliotecas de vínculo dinâmico, ou Dynamiclink library (DLL), que são programados em linguagem Visual Basic .NET ou C\#, e são adicionados ao programa através de um procedimento simples de instalação (AMES, 2006). 
Os plugins adicionados ao software de SIG desta forma podem interagir com as propriedades, funções, e objetos geográficos do MapWindow GIS ${ }^{\circ}$, permitindo que funções de geoprocessamento sejam programadas de maneira dinâmica e tendo disponível todas as funcionalidades do software, por exemplo as ferramentas de visualização, como zoom e pan, e as ferramentas de edição de legendas e alteração da simbologia.

Esta capacidade da plataforma SIG foi utilizada para o desenvolvimento deste trabalho, onde foi construída a integração entre o modelo hidrológico e o SIG através de um plugin adicionado ao MapWindow GIS®.

Para a geração de gráficos dentro do MapWindow GIS $®$ foi utilizado o plugin de uso livre ZedGraph (STEINER et al., 2005).

\section{APLICACÃO}

A Figura 01 apresenta um fluxograma geral das principais etapas de uma aplicação do modelo hidrológico MGB-IPH, destacando as partes onde a integração com SIG desenvolvida é utilizada (Interface MGB-IPH), que são as etapas de processamento de dados de entrada e pós-processamento.

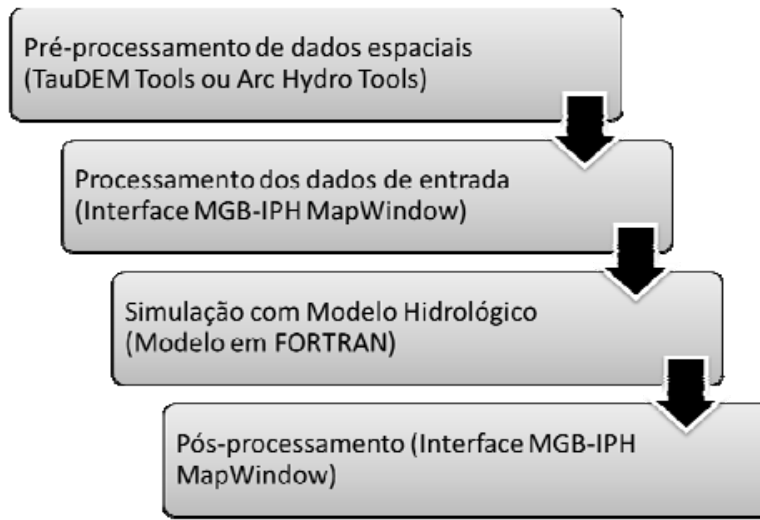

Figura 01 - Principais etapas de aplicação do Modelo Hidrológico MGB-IPH.

A seguir, a ferramenta de integração desenvolvida é apresentada simultaneamente ao desenvolvimento do estudo de caso na bacia do rio Ijuí, no estado do Rio Grande do Sul, Brasil, onde as funcionalidades do trabalho desenvolvido são comentadas e demonstradas.

\section{Simulação da Bacia do Rio Ijuí}

A Bacia Hidrográfica do rio Ijuí situa-se a norte-noroeste do Estado do Rio Grande do Sul. Ela possui área de aproximadamente $10.704 \mathrm{~km}^{2}$ e os principais cursos de água da bacia hidrográfica são os rios Caxambu, Potiribu, Conceição, Ijuizinhu e o Rio Ijuí. A Figura 02 apresenta a topografia digital e os principais cursos de água da bacia Ijuí.

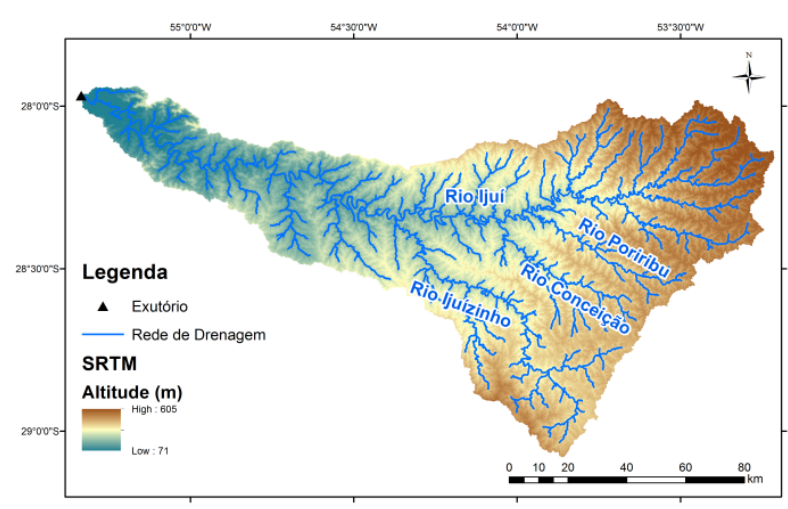

Figura 02 - Topografia e principais rios da bacia Ijuí.

A primeira etapa de aplicação do modelo MGB-IPH consiste no pré-processamento dos dados espaciais para discretização da bacia. Isto pode ser feito através das ferramentas TauDEM Tools ou das ferramentas ArcHydro Tools. Para este estudo de caso, foi utilizada a segunda opção.

Assim, a partir de um Modelo Digital de Elevação (MDE), obtido pelo projeto Shuttle Radar Topographic Mission na resolução de $90 \mathrm{mx} 90 \mathrm{~m}$ (Figura 02), foram extraídas as informações físicas e topológicas da bacia hidrográfica do rio Ijuí, como a rede de drenagem e a divisão em mini-bacias (FAN et al., 2010a). O resultado destas operações para a bacia Ijuí é apresentado na Figura 03. Ao total, a bacia foi dividida em 532 mini-bacias.

Para a definição das unidades de resposta hidrológica da bacia Ijuí foram reclassificados e processados dois mapas de solos distintos, o mapa digital de solos oriundo do projeto RADAMBrasil (Publicado em 2001 na Escala 1:5.000.000) e o mapa de solos digitalizado pela EROS Data Center/USGS (publicado em 1981 na escala 1:5.000.000) oriundo de mapeamentos de solos realizados pela Empresa Brasileira de Pesquisa Agropecuária (EMBRAPA). Também foi utilizado um mapa de vegetação com origem nas cartas-imagens de radar produzidas pelo Projeto RADAMBRASIL, que foi digitalizado e distribuído pela U.S. Geological Survey's EROS Data Center (publicado em 1992 na escala 1:5.000.000). 


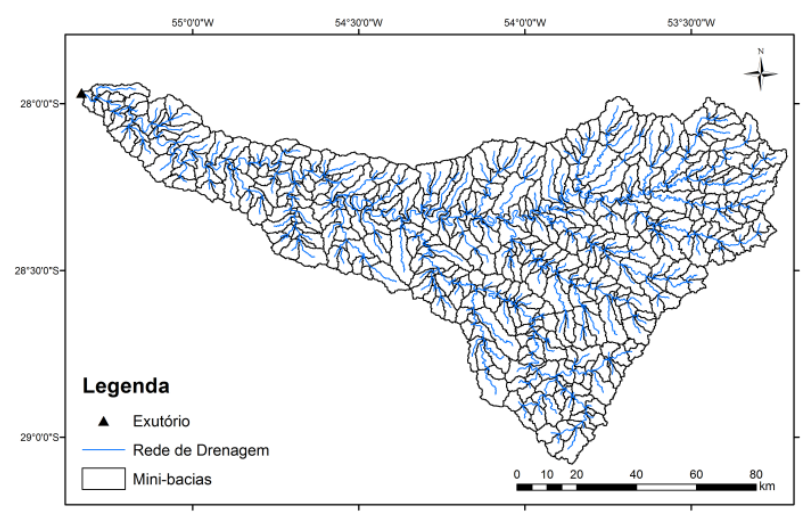

Figura 03 - Divisão em mini-bacias da bacia do rio Ijuí (Rio Grande do Sul).

Os solos foram classificados em rasos e profundos e a vegetação foi classificada em vegetação densa (florestas); campos e pastagens; e zonas de contato, resultando em 7 UHRs para a bacia (contando a UHR "água”). O mapa de UHRs da bacia é apresentado na Figura 04.

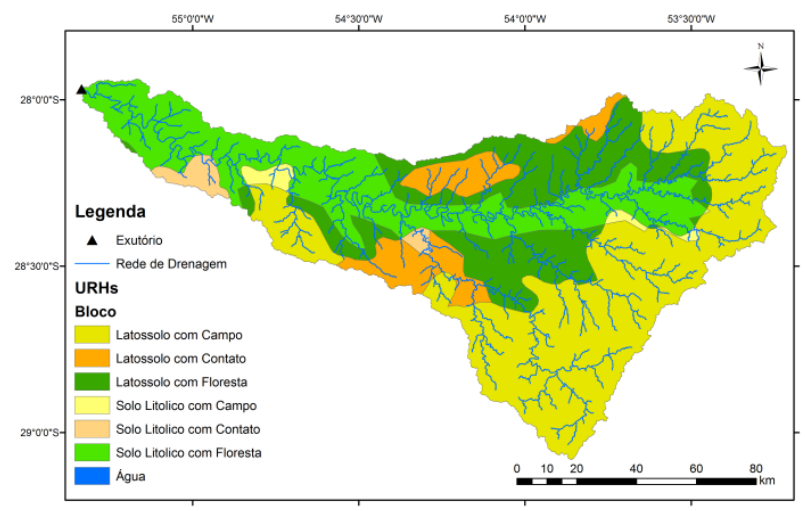

Figura 04 - Exemplo de URHs geradas para a bacia do rio Ijuí para a aplicação do modelo MGB-IPH.

Após a preparação das informações espaciais de entrada do MGB-IPH, todos os arquivos foram sumarizados e compilados em um único arquivo de entrada do modelo hidrológico (denominado MINI.MGB), através de um programa denominado PrePro_MGB, que faz parte do conjunto de ferramentas de aplicação do modelo (FAN et al., 2010b). Este arquivo gerado pelo PrePro_MGB consiste em uma tabela de atributos das minibacias que compõem a bacia hidrográfica. Entre estes atributos destacam-se a área de drenagem, as coordenadas dos centroides (calculadas através de um processo de cálculo de centro de massa para figuras planas), comprimentos e declividades, bem como a informação topológica (minibacia localizada a jusante).

Com o término da preparação dos dados espaciais de entrada do MGB-IPH, todas as demais etapas posteriores de aplicação do modelo são realizadas dentro da interface MapWindow GIS ${ }^{\circledR}$ do MGB-IPH, desenvolvida e apresentada neste trabalho. Aqui se encerra a primeira etapa do fluxograma de aplicação e começa a segunda.

A primeira tarefa para a realização de uma simulação no MapWindow GIS® é a inclusão de alguns dados espaciais gerados nas etapas anteriores no mapa e a criação de um projeto de trabalho dentro da plataforma SIG. Posteriormente, as informações gravadas no arquivo de saída do PrePro_MGB são incluídas no mapa usando a ferramenta "Gerar shapefile com MINI.MGB" da ferramenta desenvolvida neste trabalho, e a camada de informação (layer) dos centróides deve ser adicionada à área de trabalho do SIG. A Figura 05, abaixo, apresenta alguns layers, incluindo os centróides das minibacias, adicionados à interface MapWindow GIS ${ }$ do MGB-IPH.

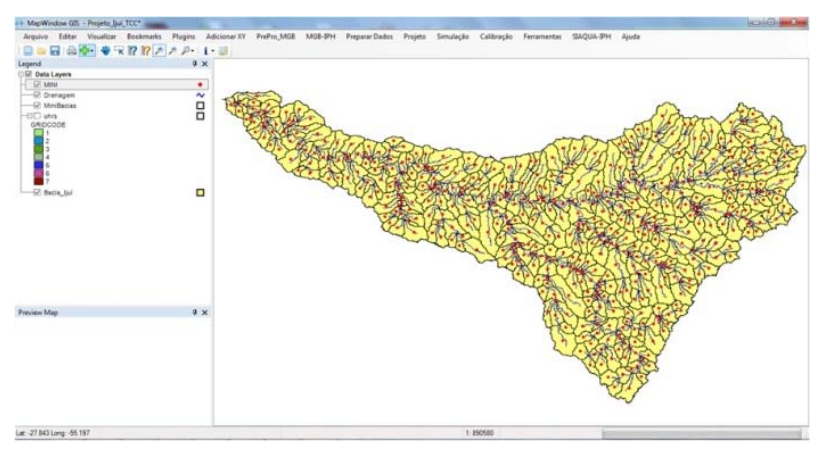

Figura 05 - Bacia do rio Ijuí na interface MapWindow GIS® do MGB-IPH, com os centróides das minibacias.

O próximo passo para a aplicação do modelo consistiu na obtenção, análise e preparação dos dados de chuva e vazão do modelo hidrológico. Para a aplicação na bacia do rio Ijuí, todos os dados de vazão utilizados para a modelagem foram obtidos no sistema Hidroweb da Agência Nacional de Águas (ANA). Para isto, foi desenvolvida uma ferramenta de importação de dados hidrológicos que permite incorporar estes dados do sistema Hidroweb da ANA. Para a bacia do rio Ijuí, foram obtidos os dados de vazão de quatorze postos fluviométricos e de trinta e seis postos pluviométricos.

Dentro da interface desenvolvida, estes dados de vazão e precipitação são analisados utilizan- 
do, respectivamente, as ferramentas "Vazão" e "Precipitação". Estas duas ferramentas são apresentadas nas Figuras 06 e 07, a seguir.

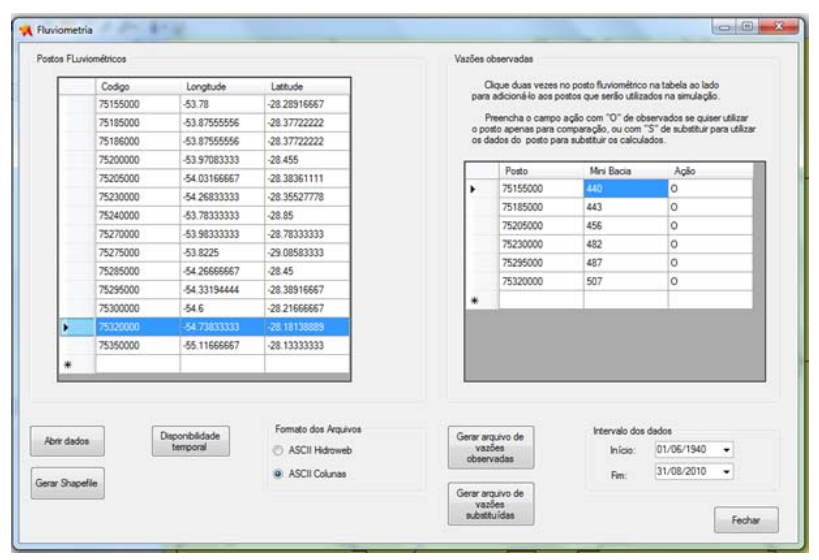

Figura 06 - Ferramenta "Vazão" da interface desenvolvida.

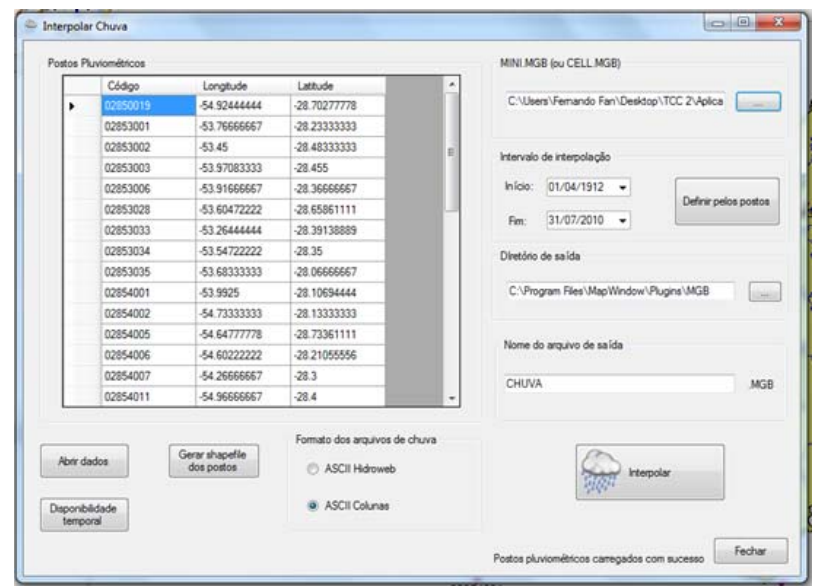

Figura 07 - Ferramenta "Precipitação" da interface desenvolvida.

Quando dados de postos fluviométricos ou pluviométricos são carregados no SIG, a interface automaticamente identifica as coordenadas de latitude e longitude de cada posto, eliminando a necessidade do usuário de inseri-las manualmente. Além disso, as ferramentas possibilitam a criação de layers com a posição dos postos, para serem visualizados no SIG, e a verificação da disponibilidade de dados em cada posto através de um gráfico do tipo de Gantt. A Figura 08 apresenta o gráfico de Gantt com a disponibilidade dos dados de vazão para a bacia Ijuí.

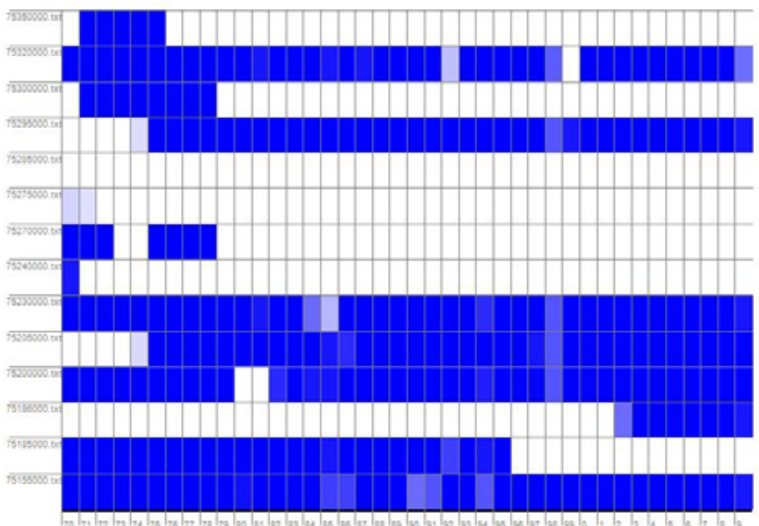

Figura 08 - Gráfico Gantt com a disponibilidade temporal de dados de vazão na bacia Ijuí.

É possível ainda, através das ferramentas desenvolvidas, acessar o hidrograma e a curva de permanência dos postos fluviométricos, e o pluviograma dos postos pluviométricos.

A partir da análise da disponibilidade temporal e espacial dos dados de chuva e vazão, são definidos quais postos de medição devem ser utilizados. Neste estudo o período para a realização da simulação foi escolhido como de 1980 a 2009, e foram gerados os respectivos arquivos de entrada do MGB-IPH.

A etapa posterior de aplicação do modelo MGB-IPH é a preparação dos dados climáticos de entrada do modelo. Para a manipulação e verificação da disponibilidade de dados de clima, que também podem ser utilizados diretamente a partir do sistema HidroWeb, é utilizada a ferramenta "Clima", apresentada na Figura 09.

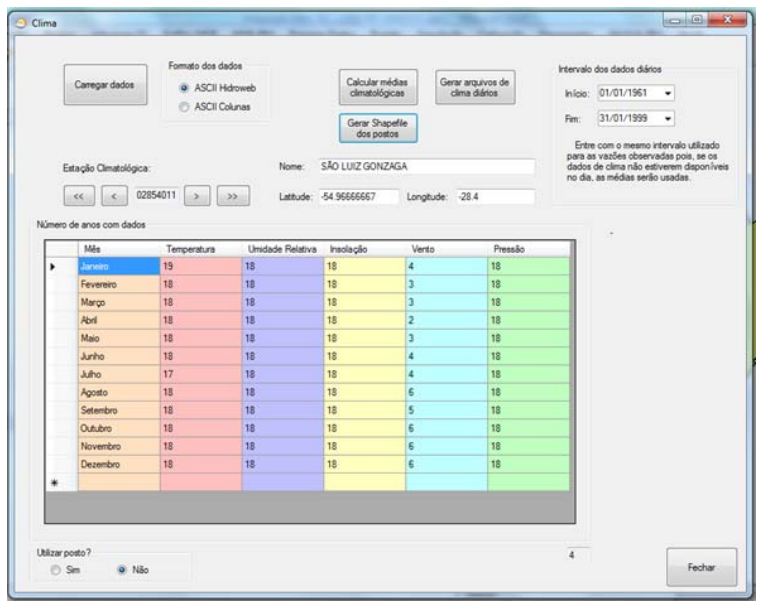

Figura 09 - Ferramenta de manejo de dados de clima da interface MapWindow GIS ${ }^{\circledR}$ do MGB-IPH. 
Os demais arquivos de entrada do modelo MGB-IPH, que são, respectivamente, descrição das URHs, Parâmetros Fixos e Parâmetros Calibráveis, são gerados também a partir de ferramentas específicas desenvolvidas para tanto. Estes dados são preparados basicamente através do preenchimento de tabelas, onde as ferramentas desenvolvidas possuem funções de apoio a esta ação. A Figura 10 apresenta o aspecto geral da ferramenta de URHs. As demais ferramentas citadas são similares.

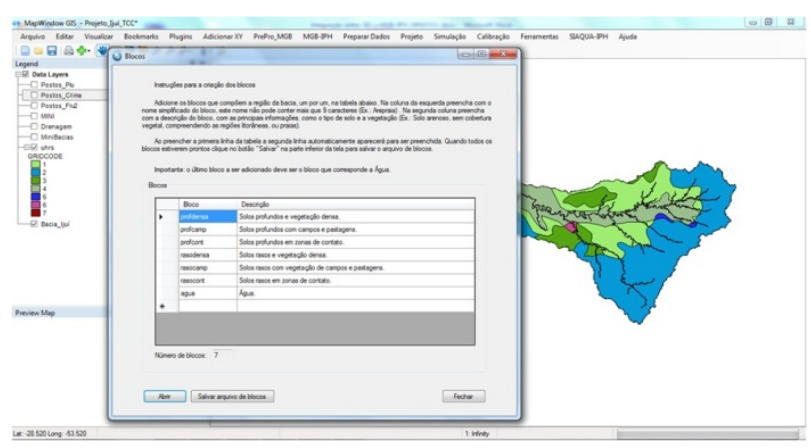

Figura 10 - Ferramenta de preparação do arquivo de entrada com a descrição das URHs.

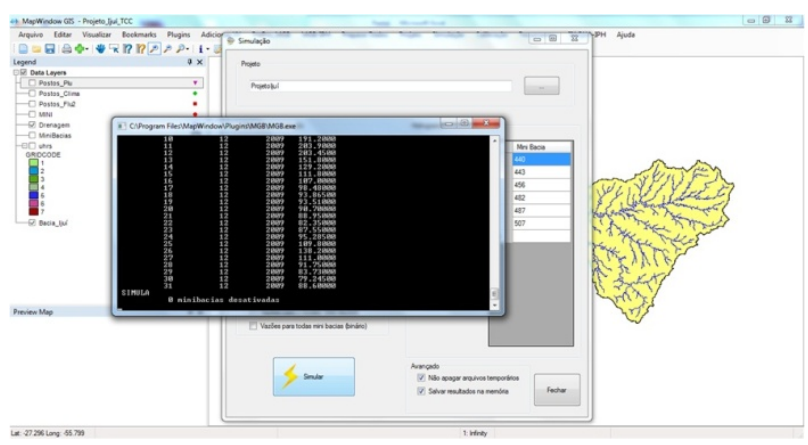

Figura 11 - MGB-IPH sendo executado via interface para a bacia Ijuí.

Após a etapa de preparação de informações de entrada, todos os dados são compilados em um projeto de simulação na interface. Com isto, se encerra a segunda etapa do fluxograma, que é a preparação de dados para a aplicação do modelo via interface.

A terceira etapa de um projeto de simulação consiste em executar o modelo hidrológico, que é programado em linguagem FORTRAN. O modelo MGB-IPH é executado a partir da ferramenta "Simulação", dentro do MapWindow GIS®. A Figura 11 apresenta o modelo MGB-IPH sendo executado via interface para a bacia Ijuí.
Ao término da execução do modelo hidrológico, se inicia a quarta etapa de um projeto de simulação, que é o pós-processamento dos resultados. Para isto, a interface desenvolvida automaticamente faz a leitura de todos os resultados da simulação. A partir deste ponto, todos os dados de saída do MGB-IPH podem ser visualizados de forma interativa dentro do SIG, através do centroide da minibacia desejada. As Figuras 12 e 13, abaixo, apresentam alguns resultados da simulação da bacia Ijuí, que foram gerados pelas ferramentas desenvolvidas.

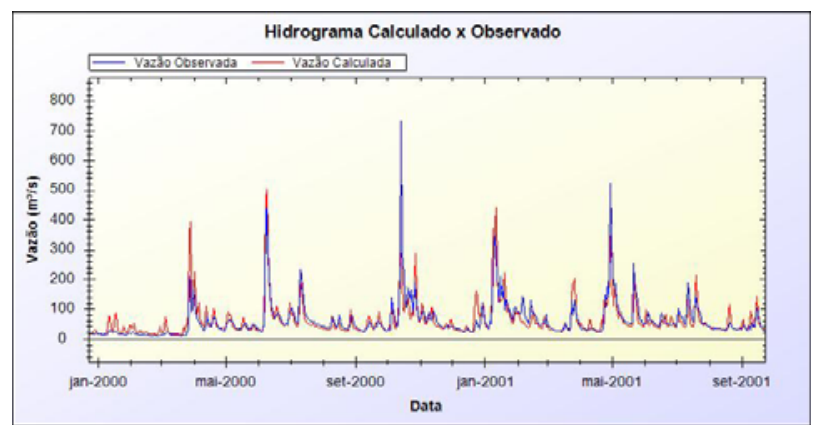

Figura 12 - resultado da simulação na mini-bacia 487, comparado ao posto fluviométrico 75295000 , na bacia Ijuí.

Além dos hidrogramas, a interface no SIG também mostra as medidas de performance do modelo quando apresenta os resultados para cada minibacia com dados fluviométricos medidos.

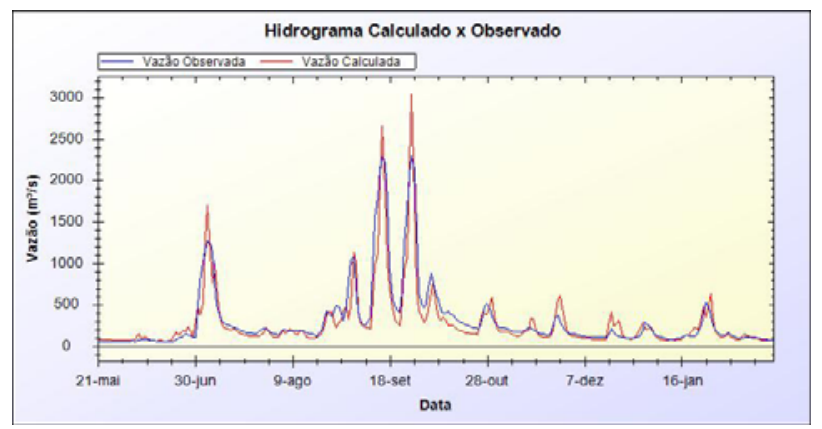

Figura 13 - Resultado da simulação na mini-bacia 507, comparado ao posto fluviométrico observado 75320000 , na bacia Ijuí.

Ainda, a interface calcula automaticamente as curvas de permanência das séries temporais calculadas pelo MGB-IPH, que podem ser acessadas via SIG. A Figura 14, abaixo, apresenta uma curva de permanência resultado da simulação para a bacia Ijuí. 


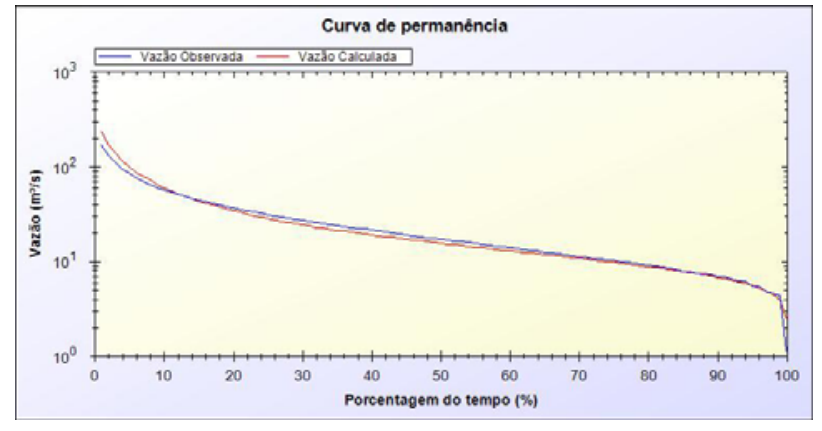

Figura 14 - Curva de permanência resultado da simulação na minibacia 456, comparado ao posto fluviométrico observado 75205000, na bacia Ijuí

\section{Ferramentas adicionais}

Além das ferramentas apresentadas no estudo de caso na bacia Ijuí, outras ferramentas foram desenvolvidas para auxiliar a aplicação do modelo MGB-IPH e para a interpretação dos seus dados de entrada e de saída.

Uma das ferramentas desenvolvidas consiste em um filtro para a determinação do escoamento de base a partir dos dados de postos fluviométricos. Este filtro também é acessado via interface SIG. A Figura 15, abaixo, apresenta um exemplo de separação de escoamento gerado via interface.

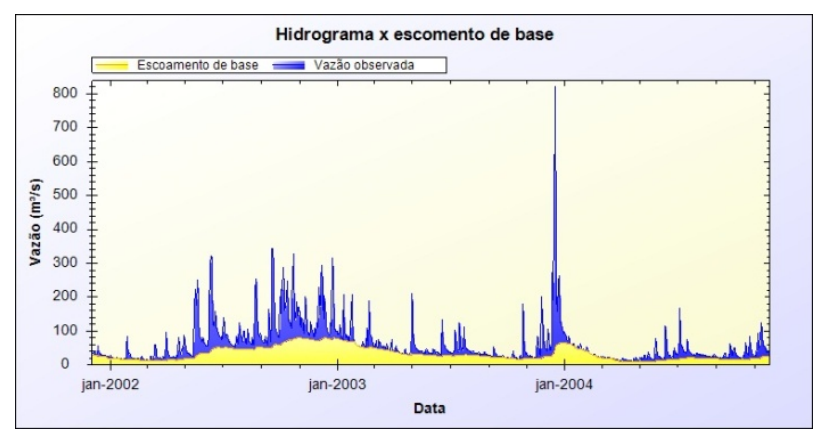

Figura 15 - Separação de escoamento elaborada via ferramenta desenvolvida.

Outra funcionalidade desenvolvida consiste em uma ferramenta de geração de dados de clima para o MGB-IPH utilizando uma base de dados interna de normais climatológicas. Esta ferramenta facilita a aplicação do modelo em locais onde dados de clima não estão facilmente disponíveis.

Por fim, o modelo MGB-IPH possui a função de calibração automática multiobjetivo, baseado no algoritmo genético MOCOM-UA (YAPO et al., 1998). Assim, como parte da integração desenvolvi- da, uma ferramenta para a preparação das informações de calibração automática também foi criada.

\section{DISCUSSÃO}

Visando potencializar o uso da simulação hidrológica, aprimorar a integração entre modelagem e as tecnologias SIG e promover desenvolvimento neste campo de estudo, o presente trabalho apresentou a concepção de uma integração entre a plataforma de SIG MapWindow GIS® e o modelo hidrológico MGB-IPH.

A integração desenvolvida entre os dois programas pode ser considerada do tipo acoplamento próximo (ou rígido), uma vez que, após a preparação dos dados de entrada do modelo hidrológico, ele é acionado automaticamente pela interface concebida, através de uma transferência interna de dados.

A ferramenta concebida foi testada na modelagem da bacia hidrográfica do rio Ijuí, e o comportamento hidrológico da bacia foi representado com sucesso.

A interface MapWindow GIS® facilita a aplicação do modelo atuando principalmente em duas fases de sua sequência de aplicação: na preparação dos dados de entrada, e na visualização e pósprocessamento dos seus dados de saída.

Na questão de preparação dos dados de entrada, a integração tem dois grandes benefícios, que são: (i) facilitar a preparação dos dados através de ferramentas que guiam o usuário ao longo do processo; e (ii) transformar este procedimento em uma atividade interativa com a visualização espacial dos dados, possibilitando uma maior compreensão do sistema simulado.

Como uma segunda forma de facilitar o uso do modelo, logo após o término da simulação, os resultados são acessados pela plataforma SIG e disponibilizados ao usuário via mapas e gráficos. Isto torna a experiência de compreensão dos resultados mais rápida e visual, já que as informações são acessadas visualizando os locais de interesse.

De uma forma geral, pode-se fazer a seguinte lista do que se acredita que sejam as principais contribuições e avanços trazidos pelo trabalho desenvolvido:

I. A criação dos dados de entrada do MGB-IPH é mais rápida e organizada, onde são gerados 
arquivos já com a estrutura de entrada do modelo MGB-IPH (não deixando margem para erros).

II. A interface possui opção de uso direto de dados provenientes do sistema HidroWeb da ANA, uma das principais fontes de dados hidrológicos sobre bacias brasileiras.

III. A interface possui ferramentas de análise de dados hidrológicos e climáticos.

IV. A interface possui uma base interna de dados de clima, que elimina dificuldades na realização de uma simulação em locais com falta destas informações.

V. Todos os resultados da simulação hidrológica são apresentados via mapas e gráficos na tela do MapWindow GIS®. Isto facilita o entendimento, a análise e a interpretação dos resultados do modelo.

Todas estas contribuições listadas trazem vantagens para a utilização do modelo hidrológico, principalmente para usuários mais inexperientes, pois as ferramentas desenvolvidas são também uma forma de aprender sobre o modelo ao mesmo tempo em se está fazendo uma aplicação.

Em relação às limitações da abordagem selecionada, pode-se argumentar que a integração do modelo com SIG exige que o MGB-IPH seja aplicado de uma forma padronizada e compatível com as ferramentas criadas no MapWindow GIS $®$. Ou seja, a aplicação por usuários avançados que desejam fazer modificações na estrutura do modelo hidrológico exige que estes abandonem a aplicação padronizada via interface e busquem diretamente o código fonte do modelo, em linguagem FORTRAN.

Todavia, esta constatação não invalida o uso da interface, que ainda pode ser usada para a preparação dos dados de entrada do modelo nestes casos.

Assim, com a ampliação do uso da integração com SIG do modelo hidrológico e a facilidade que ela traz para usuários de diferentes níveis, o MapWindow GIS® passa a ser uma forma de um usuário interagir com o modelo MGB-IPH.

Como perspectivas, espera-se que a integração apresentada neste trabalho seja utilizada para a expansão da aplicação do modelo MGB-IPH, apoiando a busca pela solução dos diversos problemas relacionados aos recursos hídricos da atualidade, e que isto sirva de motivação para o contínuo desenvolvimento desta ferramenta.

\section{DISPONIBILIDADE DO MODELO MGB-IPH}

O modelo hidrológico MGB-IPH e todas as ferramentas de acoplamento com SIG desenvolvidas e apresentadas neste trabalho encontram-se disponíveis na página do Grupo de Pesquisa em Hidrologia de Grande Escala (http://www.ufrgs.br/hge/), do Instituto de Pesquisas Hidráulicas (IPH), da Universidade Federal do Rio Grande do Sul (UFRGS).

\section{AGRADECIMENTOS}

Os autores deste artigo agradecem aos revisores anônimos que deram importantes contribuições para a qualidade do trabalho.

\section{REFERÊNCIAS}

AMES, D. P. Getting Started with the MapWinGIS ActiveX Control. EUA, 2006.

AMES, D. P., C. MICHAELIS, A. ANSELMO, L. CHEN, AND H. DUNSFORD, 2008. MapWindow GIS. Encyclopedia of GIS. New York, Sashi Shekhar and Hui Xiong (eds). Springer, 633-634.

BEVEN, M.; KIRKBY, M.J. A physically based, variable contributing and model of basin hydrology. Hydrological Sciences Bulletin, 24: 43-69, 1979.

BURROUGH, P.H.A. Methods of spatial analysis in GIS, International Journal of Geographical Information Systems, 4(3), 221-223, 1990.

COELHO, G. B., Análise e simulação do processo de transformação de chuva em vazão com suporte de Sistema de Informações Geográficas (SIG); 2006; 81f. Dissertação de mestrado em Ciências Geodésicas e Tecnologias da Geoinformação. Universidade Federal de Pernambuco Centro de Tecnologia e Geociências. Recife, 2006.

COLLISCHONN, W. ; ALLASIA, D. G. ; SILVA, B. C. ; TUCCI, C. E. M. The MGB-IPH model for largescale rainfall-runoff modelling. Hydrological Sciences Journal, v. 52, p. 878-895, 2007. 
COLLISCHONN, W., TUCCI, C. E. M. Simulação hidrológica de grandes bacias. Revista Brasileira de Recursos Hídricos, v. 6, n. 2, 2001.

ESRI, 2007. Arc Hydro Tools version 1.2 for ArcGIS 9.2 Tutorial. California. 110 p.

FAN, F. M.; COLLISCHONN W.; BUARQUE, D. C.; PAIVA, R. C. D. Projeto Amazônia. IPH - UFRGS. Manual Arc-HYDRO para aplicação do modelo MGB-IPH. Versão 1.2. p. 65, 2010a.

FAN, F.M.; BUARQUE, D.C.; COLLISCHONN, W.; PAIVA, R.C.D.; KAYSER, R. B. Manual do PreProMGB para aplicação do modelo MGB-IPH. Projeto Integrado de Cooperação Amazônica e de Modernização do Monitoramento Hidrológico, FINEP/ANA/IPH-UFRGS, Porto Alegre (RS), p. 17, 2010b.

FERRAZ，F.F.B.; MILDE， L.C.E.; MORTATTI，J. Modelos Hidrológicos Acoplados a Sistemas de Informações Geográficas: um Estudo de Caso. Revista de Ciência e Tecnologia. 14 - pp.45-56, 1999.

GEORGE, C. e LEON , L.F. WaterBase: SWAT in an open source GIS, The Open Hydrology Journal, Bentham Science Publishers Ltd., Vol. 2, pp.1-6, 2008.

GETIRANA, A. C. V. ; Bonnet, M.-P. ; ROTUNNO FILHO, O. C. ; COLLISCHONN, W. ; GUYOT, J.-L. ; Seyler, F. ; MANSUR, W. J. Hydrological modelling and water balance of the Negro River basin: evaluation based on in situ and spatial altimetry data. Hydrological Processes. V. 24, Ed. 22, p. 3219-3236, 2010.

GOODCHILD, M. F.; HAINING, R.; WISE, S. Integrating GIS and spatial data analysis: Problems and possibilities. International Journal of Geographical Information Systems, 6, p. 407- 423, 1992.

GOODCHILD, M.F. The state of GIS for environmental problem-solving. In Goodchild, M.F., Parks, B.O. and Steyaert, L.T. (ed.), Environmental Modeling with GIS. Oxford University Press, Oxford, 8-15, 1993.

HESSION, C.W. e V.O. SHANHOLTZ. A Geographic Information System for Targeting Nonpoint Source Agricultural Pollution. Journal of Soil and Water Conservation 43:264-266, 1988
LIAO, H. e U.S. TIM. An Interactive Modeling Environment for Nonpoint Source Pollution Control. Journal of the American Water Resources Association (JAWRA) 33(3):591-603, 1997.

MAIDMENT, D. (editor), 2002. Arc Hydro - GIS for Water Resources, ESRI Press, Redlands, CA.

MARTIN, P. H.; LEBOEUF, E. J.; DOBBINS, J. P.; DANIEL, E. B.; ABKOWITZ, M. D. Interfacing GIS with water resource models: A state-of-the-art review. Journal of the American Water Resources Association. December pp. 1471-1487, 2005.

MELO, H. A., CUNHA, J. B. L., NÓBREGA, R. L. B., RUFINO, I. A. A. e Galvão, C. O. Modelos Hidrológicos e Sistemas de Informação Geográfica (SIG): Integração possível. IX Simpósio de Recursos Hídricos do Nordeste, 2008.

MIZGALEWICZ, P.J \& MAIDMENT, D.R.(1996). Modeling Agrichemical Transport in Midwest Rivers Using Geographic Information Systems. CRWR Online Report 96-6, Center for Research in Water Resources, University of Texas, Austin, http://www.cnwr.utexas.edu/online.html, consulta em Junho de 2010.

MOORE, I. D. (1996). Hydrologic modeling and GIS. In M. F. Goodchild, B. O. Parks, e L. T. Steyaert (Eds.), GIS and environmental modeling: Progress and research issues (pp. 143-148). Fort Collins, CO: GIS World Books.

MOORE, I.D., R.B. GRAYSON, e G.J. BRURSCH. A Contour- Based Topographic Model for Hydrological and Ecological Applications. Earth Surface Processes and Landforms 13:305-320, 1988.

NYERGES, T. Geographic Information Abstractions: Conceptual Clarity for Geographic Modeling. Environment and Planning A, vol. 23: p. 1483-1499, 1991.

OLIVERA, F., M. VALENZUELA, R. SRINIVASAN, J. CHOI, H. CHO, S. KOKA, and A. AGRAWAL. ArcGIS-SWAT: A geodata model and GIS interface for SWAT. J. American Water Resour. Assoc.42(2): 295-309, 2006.

OLIVIERI, L.J., G.M. SCHAAL, T.J. LOGAN, W.J. ELLIOT, e B. MOTCH. Generating AGNPS Input Using Remote Sensing and GIS. Paper 91-2622, 
American Society of Agricultural Engineers, St. Joseph, Michigan, 1991.

PAIVA, R. C. D., Modelagem hidrológica e hidrodinâmica de grandes bacias: Estudo de caso na bacia do rio Solimões. 2009. Dissertação (Mestrado em Recursos Hídricos e Saneamento Ambiental) - Universidade Federal do Rio Grande do Sul, Financiadora de Estudos e Projetos. Pg. 182.

PAZ, A. R.; BUARQUE, D C.; COLLISCHONN, W.; VICTORIA, D. C.; ANDRADE, R. G.; Discretização de Modelos Hidrológicos de Grandes Bacias: grade regular x mini-bacias. XIX Simpósio Brasileiro de Recursos Hídricos. p. 18, 2011

PEREIRA, M. M. E. 2010. Integração de Modelos Hidrológicos e SIG para ferramentas de Suporte à tomada de decisões. Dissertação de Mestrado. IPHUFRGS.

STEINER JEAN L., CHEN JIN-SONG E SADLER JOHN. STEWARDS: An Integrated Data System for ARS Watershed Research. Proceedings of Oklahoma Water 2005, Tulsa, OK, September 27 and 28, Paper \# 11 Oklahoma Water Resources Research Institute, Stillwater, p. 10, 2005;

SUI D. Z.; MAGGIO R. C. Integrating GIS with hydrological modeling: practices, problems, and prospects. Computers, Environment and Urban Systems. p. 35-51, 1999.

TARBOTON, D. G. 2002. "Terrain Analysis Using Digital Elevation Models (Taudem),"Utah Water Research Laboratory, Utah State University. Acessado em Maio de 2013: http://www.engineering. usu.edu/dtarb.

TIM, U.S., S. MOSTAGHIMI, e V.O. SHANHOLTZ, Identification of Critical Nonpoint Pollution Source Areas Using Geographic Information Systems and Water Quality Modeling. Water Resources Bulletin 28:877-887, 1992.

TUCCI, C. E. M. Modelos hidrológicos. Ed. Universidade/ UFRGS, Porto Alegre, p. 669, 1998.

USACE, "Geospatial hydrologic modeling extension: HEC-GeoHMS user's manual”. U.S. Army Corps of Engineers, Hydrologic Engineering Center, Report CPD-77, 2009.
WHITEAKER, T.; MAIDMENT D. R. Geographically Integrated Hydrologic Modeling Systems. Dissertation. The University of Texas at Austin, 2004. CRWR Online Report. Acessado em Maio de 2013: $<$ http://www.crwr.utexas.edu/reports/2004/rpt044.shtml $>$.

YAPO, P. O.; GUPTA, H. V.; SOROOSHIAN, S. Multi-objective global optimization for hydrologic models. Journal of Hydrology Vol. 204 pp. 83-97, 1998.

ZEILHOFER, P. et al. Um ambiente SIG para modelagem integrada da qualidade da água utilizando QUAL2E. Caminhos da Geografia - Revista on line, 8 (10) 107-125, setembro. Instituto de Geografia UFU, 2003.

\section{Integration Of Model MGB-IPH With The Geo- graphic Information Systems}

\section{ABSTRACT}

Problems involving efficient use of water resources are major challenges to be faced by humanity in the coming decades. In this context, hydrological modeling application is an extremely useful tool to develop assessments and to support decision-making. The distributed type hydrologicalal models can represent the behavior of a watershed in a complex manner, allowing detailed analysis and studies based on their results. One of the essential requirements for the use of distributed models is the detailed physiographic description of the studied basin, obtained by using Geographic Information Systems (GIS) platforms. Aiming to enhance the use of hydrological simulation and to develop solutions for proper water resources management, this paper presents the design of a coupling between the MapWindow GIS® platform and the MGB-IPH hydrological model. The developed integration can be considered a close coupling, due to the internal data transfer characteristics developed. The designed tool was successfully tested for the modeling of the Ijui River basin (Southern Brazil). The application showed that a high degree of integration was obtained between the GIS and the hydrological model. Useful tools were created for input data handling and representation in the GIS environment, and for post-processing and analysis of the hydrological model results.

Key-words: hydrological model, geographic information system, MGB-IPH. 